\title{
Deployment of Query Validation for Finite Range Query Scheme in Wireless Sensor Networks
}

\author{
S.Kumaravel \\ Research Scholar, Manav Bharti University, Solan (Hp)
}

\begin{abstract}
Adversary attack on wireless sensor network is a very major issue on wireless sensor networks deployment. Adversaries send the replica node as same as like original node to capture all necessary information and the key values. Deployment of a wireless sensor network (WSN) system is a significant step since hypothetical models and hypothesis habitually vary from genuine environmental uniqueness and presentation at the operation site. In addition, such systems are regularly situated in areas that are complicated to achieve or even unreachable for definite periods of time. The previous work provided a replica detection scheme with Finite Range Query (FRQ) technique to effectively recognize the mobile replica nodes (acting as adversary) and eradicate the varying query ranges of mobile sensor nodes. But it is necessary to validate the query to improve the detection of mobile replica nodes. Therefore, it is essential to validate the functionality of the scheme at the time of the operation, thus reducing the risk of untimely failures. Unintentionally, the validation reduces the cost of revisiting the position in the near prospect for re-deployment, preservation, or repairs. In this paper we present a deployment query validation framework DQV that consists of procedures for WSN status assessment and verification. DQV is supported and has demonstrated the potential for early problem detection at three levels of WSN validation: wireless network physical and logical integrity, sensor node devices, and connectivity to the backend such as a data server over the Internet. The
\end{abstract}

DQV framework will validate the query at each step taken in the Wireless sensor networks. An experimental evaluation is conducted to estimate the performance of the proposed deployment of query validation for finite range query scheme in WSN [DQV-FRQ] in terms of bandwidth, delay, data loss.

Keywords: Wireless sensor networks, Mobile replica nodes, Finite range query, Query validation scheme.

\section{INTRODUCTION}

The current increase and extensive acceptance of wireless networking skills for mobile communication applications has glowed frequent visualization of an eternally more networked and interactive world. In wireless sensor networks, wireless communication and calculating elements are shared with incorporated sensors to facilitate firmly joined interaction with the corporeal world. As a novel request province for wireless expertise, key confronts here are (i) the restricted possessions of the greatly incorporated nodes that are to be leveraged by the sum of procedure organized and the association among them, (ii) the rigid combination of application, nodes and the atmosphere and (iii) the wide practice profile by systems-experts and non-expert users similarly.

Wireless sensor networks have been established with the primary endeavor of supervising a occurrence (sensing) and performing procedures in response (actuation). The architectural principle of such networks is a collection of system sensors, entrenching a detector and an 
electromechanical (actuating) appliance, being jointly proficient of monitoring, determining, responding and conversing on a trend and distributing it with each others. To obtain practical observations and present an enough reaction, numerous detectors and actuators may be mandatory, each giving a fundamental examination and the equivalent reaction (e.g., a hotness sensor and a temperature element), which may be united to present a comprehensive behavior. With sensors, the solution restriction remains resource saving. It has provided as the chief dispute for mounting specific memory, energy, low-data-rate protocols, computationallyaware, systems and requests that jointly endeavor to broaden nodes lifetime.

The assumption and framework of wireless sensor networks (WSN) have developed commendably leading to exploitation of appealing and important examining systems. Nevertheless, the presentation of the systems after the operation frequently changes significantly from the projected or practical at the time of the development.

Owing to the unattended environment of wireless sensor networks, an opponent can detain and compromise sensor nodes, construct replicas of them, and then increase a selection of attacks with these replicas. These replica node attacks are hazardous since they permit the aggressor to influence the cooperation of a few nodes to exercise organize over much of the network.

In this work, mobile replica nodes are identified by the finite range query scheme and the process of enhancing the query validation process carries over in this work by introducing a Deployment of query validation framework to enhance the process of wireless sensor networks.

\section{LITERATURE REVIEW}

Owing to the unattended environment of wireless sensor networks, an opponent can confine and cooperate sensor nodes, construct replicas of them, and then increase a mixture of attacks with this reproduction. These duplicate node assaults are hazardous since they permit the assailant to influence the cooperation of a few nodes to exercise control over much of the network. Numerous replica node recognition schemes have been planned in the literature to preserve beside such attacks in fixed sensor networks. In [1], we suggest a rapid and efficient mobile replica node discovery system using the Sequential Probability Ratio Test.

We believe the position examination in a mobile ad-hoc network (MANET), where every node needs to continue its position information. It extends a stochastic chronological decision structure [2] to evaluate position update resolution trouble. Deployment of a wireless sensor network (WSN) system is a dangerous pace because hypothetical models and hypothesis habitually diverge from genuine ecological uniqueness and presentation at the operation site. A deployment time validation framework [3] SeeDTV comprises of system and measures for WSN status estimation and confirmation.

For wireless sensor network, a control duty based approach is used [4] to manage the duty series during the queue organization to facilitate high performance beneath changeable traffic rates in a liberated localization process [10]. When nodes operate in a duty cycling mode, path routine can be enhanced if the objective action can be calculated and nodes beside the course can be proactively awakened. Probability-based Prediction and Sleep Scheduling protocol (PPSS) [5] used to progress energy effectiveness of positive wake-up.

To retrieve the data collection from wireless sensor networks, tree based structural system is being used [6]. An unnecessary packet collision leads to envelope losses and retransmissions, resulting in noteworthy transparency costs and latency. In order to deal with this issue, we initiate a disseminated and scalable scheduling access system for objective detection process [9] that diminishes high data loss in data- 
intensive sensor networks [7]. For target detection, distributed energy optimization scheme is also being utilized [11] with probability prediction and sleep scheduling algorithm [12].

Energy constraint is an imperative concern in wireless sensor networks. This paper [8] proposes a dispersed energy optimization system for object tracking applications. Sensor nodes are gathered by the greatest entropy clustering. Then, the sensing pasture is spitted for similar sensor deployment optimization. From the work, we have seen that the proposed validation scheme used with query detection process in wireless sensor networks.

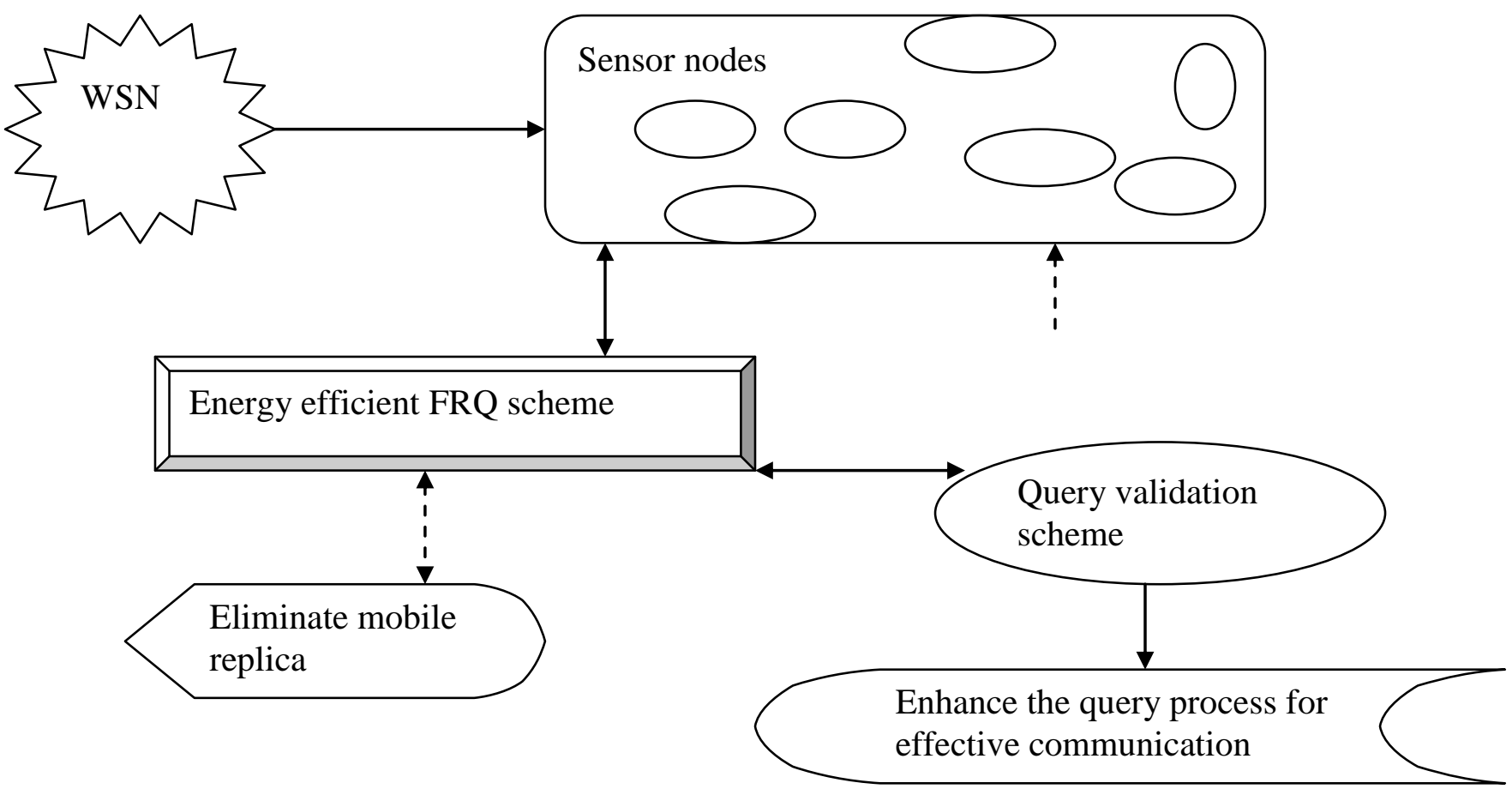

Fig 1. Proposed deployment of query validation for finite range query scheme in WSN 


\section{PROPOSED DEPLOYMENT OF QUERY VALIDATION SCHEME IN WSN}

The proposed work is efficiently designed for enhancing the query validation process in continuation with an existing identification of mobile replica nodes in finite range query scheme. By validating the query in wireless sensor networks, the mobile replica nodes are removed entirely and it reduces the loss of data occurred while transaction between the nodes in the network environment. The query validation process is carried over here using the deployment of query validation framework. The proposed deployment of query validation for finite range query scheme in WSN [DQV-FRQ] processed based on two operations. The first operation describes about the process of identification of mobile replica nodes in WSN. The second process is to apply the deployment of query validation scheme to enhance the query operation of identifying the mobile replica nodes in WSN. The architecture diagram of the proposed deployment of query validation scheme in WSN is shown in fig 1.

The first process describes about the identification of mobile replica nodes using the finite range query scheme. The mobile replica nodes are computed based upon the nodes movement in the wireless sensor networks. It efficiently recognizes the mobile replica nodes (acting as adversary) and removes the unreliable query varieties of mobile sensor nodes. Besides energy efficiency of the sensor nodes are enhanced by diminishing the message query communication on data aggregation.

The first process describes about the identification of mobile replica nodes using the finite range query scheme. The mobile replica nodes are computed based upon the nodes movement in the wireless sensor networks. It efficiently recognizes the mobile replica nodes (acting as adversary) and removes the unreliable query varieties of mobile sensor nodes. Besides energy efficiency of the sensor nodes are enhanced by diminishing the message query communication on data aggregation.

The second process describes the process of enhancing the query validation scheme in the occurrence of mobile replica nodes in the network environment. The query validation scheme is processed on three different levels as wireless network physical and logical integrity, sensor node devices, and connectivity to the back-end such as a data server.

\subsection{Identification of replica nodes in WSN using FRQ}

The Finite Range Query Scheme is used in the previous work made to endeavor the mobile duplication detection problem whereas we construct an arbitrary pace with two restrictions in such a way that each pace is dogged by the research rapidity of a mobile node. The lesser and higher limits will be configured to be associated with speeds below and in burden of Vmax, likewise. Every time a mobile sensor node schedules to a new location, each one of its neighbors desires for a obvious state having its position and time information and decides possibility of whether to promote the received state to the base station. The base station evaluates the speed from all two consecutive states of a mobile node and accomplishes the Energy Efficient Finite Range Query Scheme by permitting for speed as a practical model. Once the base station makes a decision that a mobile node has been replicated, it eliminates the replica nodes from the network.

\subsection{Deployment of query validation scheme}

In this subsection, we briefly explain how the validation process takes place using FRQ works. Assuming the process of operating a WSN with the Finite Range query and the validation process is defined as inspecting the circumstances of the organized sensor system at three levels: individual nodes, network communications, and the entire system counting the link to the exterior data services. 
During the deployment, queries are used to support the entire network transaction. For example, query validation scheme aids in testing individual parts of each node, such as the data transmission, data receiver, and retrieval process. If all these components perform well, we consign a local ID to this node and attach it to the network. For example, the localization of the nodes in the wireless sensor networks may decide their IDs dependent on their location. After validating key aspects of the functionality of each node we check the message link excellence among the nodes.

The validation of query is processed at three stages of communication in wireless sensor

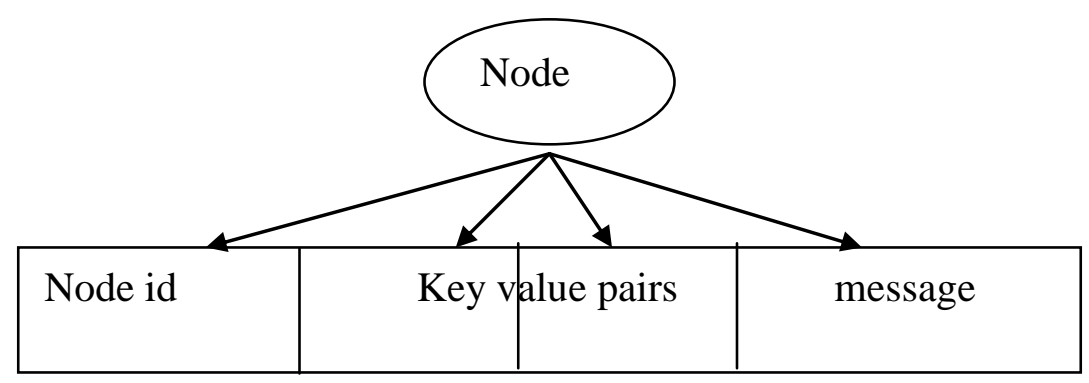

Fig 2. Node validation scheme by DQV

After verifying with the nodes present in the wireless sensor network, the valid nodes are available. The node communication is done based on sending and receiving the query and outcome with the free nodes for passing the message from source to destination. For sending the message, the sending query must contain a valid node id which is given by the authorized entity and the key value pairs for sharing the message with the destination. Before delivering the message, it is necessary to check the destination node id by the query validation scheme. The Deployment query validation scheme openly communicates with every node to authenticate the packet transmission and link feature by inspecting the node id, key value pairs provided by the authorized entity. It establishes the action of every node based on its contribution in the communications. network environment. The first stage of query processing is made with the identification of original node in the network environment. Even the finite range queries are used for the removal of mobile replica nodes, there is a great extent of nodes to be extracted or discarded by the attacker. At this sense, it is necessary to validate the query by identifying the node authorized id given by the certified authority. The node identification query is processed based on node id, key value pairs. The process of query validation for nodes present in the sensor network is described in fig 2.
If a node is hardly ever transmitting or, on the converse, persistently retransmitting, it may point out a crisis owing to the node or its location. Finally, deployment of query validation observes the entire system to authenticate that it has a least point of capacity as defined by the scrupulous application. For instance, deployment of query validation scheme checks the sample queries, and commences system supports these definite features to be contacted and influenced.

In other case, the query validation is also being done when more number of queries is engaged in a queue. The query will be in that queue till its validity over the queries gets over. The validation is based on, as said above, node id, key value pairs. Until the key is on activation, the query has been processed at any time. Once 
the key exceeds the activation process, it discards and removed from the query processing list. The algorithm below describes the over all query validation scheme in a chronological manner as follows:

Step 1: Set of nodes

Step 2: For each node

Step 3: $\quad$ Assign node id

Step 4: End for

Step 5: Using Finite Range Query,

Step 6: Identify the replica mobile node

Step 7: End

Step 8: For each node

Step 8: Validate finite query in means of (based on node id, key value pairs)

Step 9: Sensor node detection

Step 10: Server connections

Step 11: End For

Step 12: End

Using the above algorithm, the entire process of the proposed query validation scheme is processed. The query validation process is done based on number of queries processed by each sensor nodes. Once the key is invalid, the node is discarded from the list. Otherwise it continues its process until the query achieves the results.

\section{EXPERIMENTAL EVALUATION}

We simulated the proposed deployment of query validation scheme in a mobile sensor network by using the ns-2 network simulator. In the simulations, we set up $\mathrm{n}$ nodes consistently at arbitrary surrounded by a $900 \times 900$ square, with n changeable among 100 and 1000 . We determine the mobile sensor node movement patterns. In particular, to exactly estimate the presentation of the system, we use the RWM model in which each node progress to an arbitrarily selected position with an arbitrarily chosen speed among a predefined minimum and maximum speed.
We guess the standard unit disc bidirectional communication representation and we change the message range, so that every node will include roughly 40 neighbors on average. The moving mobile sensor networks stays there for a predefined pause time. After the pause time, it then randomly chooses and moves to another location. This arbitrary progression is constant during the simulation period. All simulations were performed for 1,000 simulation seconds. We fixed a pause time of 20 simulation seconds and a minimum moving speed of $1.0 \mathrm{~m} / \mathrm{s}$ of each node. Each node uses IEEE 802.11 as the medium access control protocol in which the transmission range is $50 \mathrm{~m}$. To emulate the speed errors caused by the inaccuracy of time synchronization and localization protocols, we modify the measured speeds with maximum speed error rate. The performance of the proposed deployment of query validation for finite range query scheme in WSN is measured in terms of
i) Bandwidth,
ii) Delay,
iii) Data loss.

Bandwidth is regularly termed as data transfers rate - the quantity of data that can be conceded from one point to another in a specified time period (typically a second).

The delay of a network identifies how long it receives for a bit of data to move transversely the network from one node to another. It is typically considered in multiples or fractions of seconds.

\section{RESULTS AND DISCUSSION}

In this work, we have seen the process of query validation scheme for the queries which have to be run put using the Finite Range Query effectively discover the mobile replica nodes and enhancing the query validation process to obtain an accurate query outcome. The below table and graph describes the performance of the 
proposed deployment of query validation for finite range query scheme in WSN and compared with an existing Finite Range Query scheme for detecting mobile replica nodes.

\begin{tabular}{|c|c|c|}
\hline \multirow{2}{*}{ No. of data } & \multicolumn{2}{|c|}{ Bandwidth (bps) } \\
\cline { 2 - 3 } & Proposed DQV & Existing FRQ \\
\hline 10 & 15 & 10 \\
\hline 20 & 25 & 15 \\
\hline 30 & 36 & 20 \\
\hline 40 & 45 & 24 \\
\hline 50 & 60 & 30 \\
\hline
\end{tabular}

Table 1. No. of data vs. Bandwidth

The above table (table 1) describes the bandwidth consumption when more number of data increases in the wireless sensor network environment. The outcome of the proposed deployment of Query Validation Scheme in WSN is compared with an existing Finite Range Query for detecting mobile replica nodes. query processing scheme will be more effective than the existing FRQ which only processed the query without any validation process. The bandwidth rate is measured in terms of number of data that can be transferred in a second i.e., in terms of bps (bits per second). Compared to an existing FRQ, the proposed provides a high bandwidth rate by passing the data from one node to another node in a given short interval of time. The query validation process is done based on the given query scheme like retrieving data, sending and receiving data and so on. The proposed DQV outperforms well in terms of bandwidth rate and variance is $30-40 \%$ high contrast to an existing FRQ scheme.

Table 2. No. of data vs. Delay

\begin{tabular}{|c|c|c|}
\hline \multirow{2}{*}{ No. of data } & \multicolumn{2}{|c|}{ Delay (sec) } \\
\cline { 2 - 3 } & Proposed DQV & Existing FRQ \\
\hline 10 & 5 & 10 \\
\hline 20 & 7 & 18 \\
\hline 30 & 9 & 29 \\
\hline 40 & 12 & 34 \\
\hline 50 & 15 & 48 \\
\hline
\end{tabular}

Fig 3 describes the

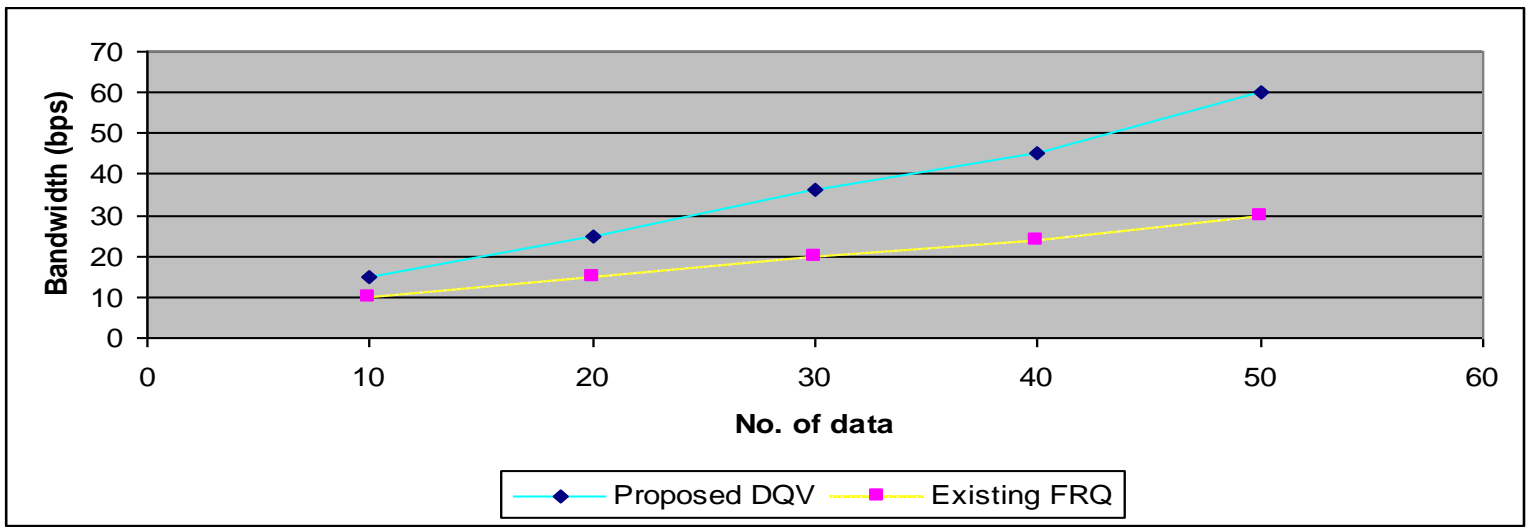
number of data present in the wireless sensor network environment. Since the proposed DQV followed the query validation scheme process, the 
Fig 3. No. of data vs. Bandwidth

The above table (table 2) describes the delay occurred when more number of data increases in the wireless sensor network environment. The outcome of the proposed deployment of Query Validation Scheme in WSN is compared

with an existing Finite Range Query for detecting mobile replica nodes.Fig 5.2 describes the occurrence of delay raised over the data which are ready to pass onto the network. The proposed deployment of Query validation scheme performed the validation scheme efficiently in terms of node key value pairs, id and transmit message. Since the validation scheme is carried over with the wireless sensor network, the delay in transmission is less. The delay is measured in terms of seconds (secs) meant that a bit of data that can be transmitted over the network in a less interval of time. Compared to an existing Finite Range Query scheme which process and transmits the network by enhancing the query, in this, there is a great extent of delay if more number of queries is waiting in the queue for transmission. But in the proposed DQV, the validation scheme taken place to validate the query and it allow only the validated query to process on the network, otherwise it discarded. The delay is low in the proposed deployment of Query Validation Scheme in WSN.

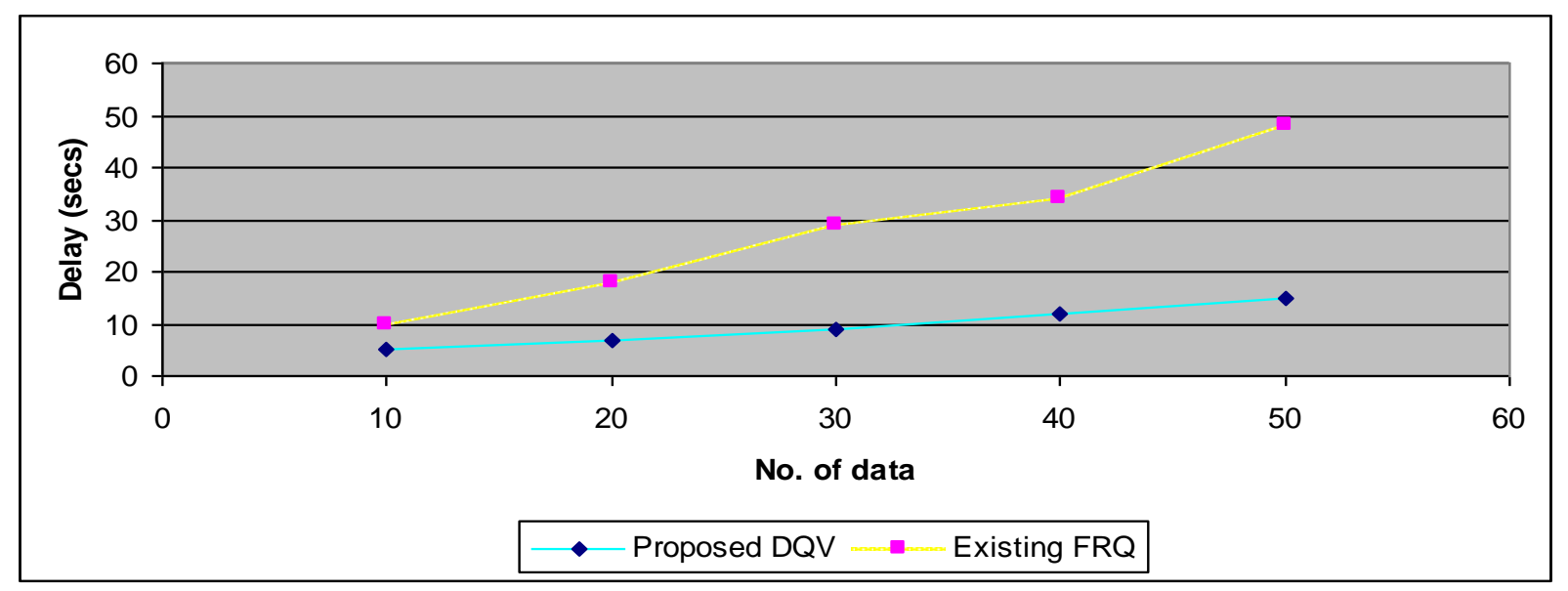

Fig 4. No. of data vs. Delay 
Table 3. No. of data vs. Data loss

\begin{tabular}{|c|c|c|}
\hline \multirow{2}{*}{ No. of data } & \multicolumn{2}{|c|}{ Data loss } \\
\cline { 2 - 3 } & Proposed DQV & Existing FRQ \\
\hline 10 & 2 & 9 \\
\hline 20 & 5 & 18 \\
\hline 30 & 9 & 25 \\
\hline 40 & 13 & 36 \\
\hline 50 & 16 & 42 \\
\hline
\end{tabular}

The above table (table 3) describes the loss of data obtained when more number of data increases in the wireless sensor network environment. The outcome of the proposed deployment of Query Validation Scheme in WSN is compared with an existing Finite Range Query for detecting mobile replica nodes.

Fig 5 describes the data loss occurred when number of data increases in the wireless sensor networks. The data loss in the wireless network is high when we use the finite range query scheme. If the number of query processing exceeds the limit, then the chance of losing the data from the wireless sensor network is high. To protect the data from lost, in this work we used the query validation scheme to validate the query and formed the query processing more effective. Compared to an existing Finite Range Query for detecting mobile replica nodes, the proposed deployment of Query Validation Scheme in WSN outperforms well.

At last, it is being concluded that the proposed deployment of Query Validation Scheme in WSN efficiently validate the query based on the presence of data. The validation is performed based on the node key value pairs, node id based on

prescence.

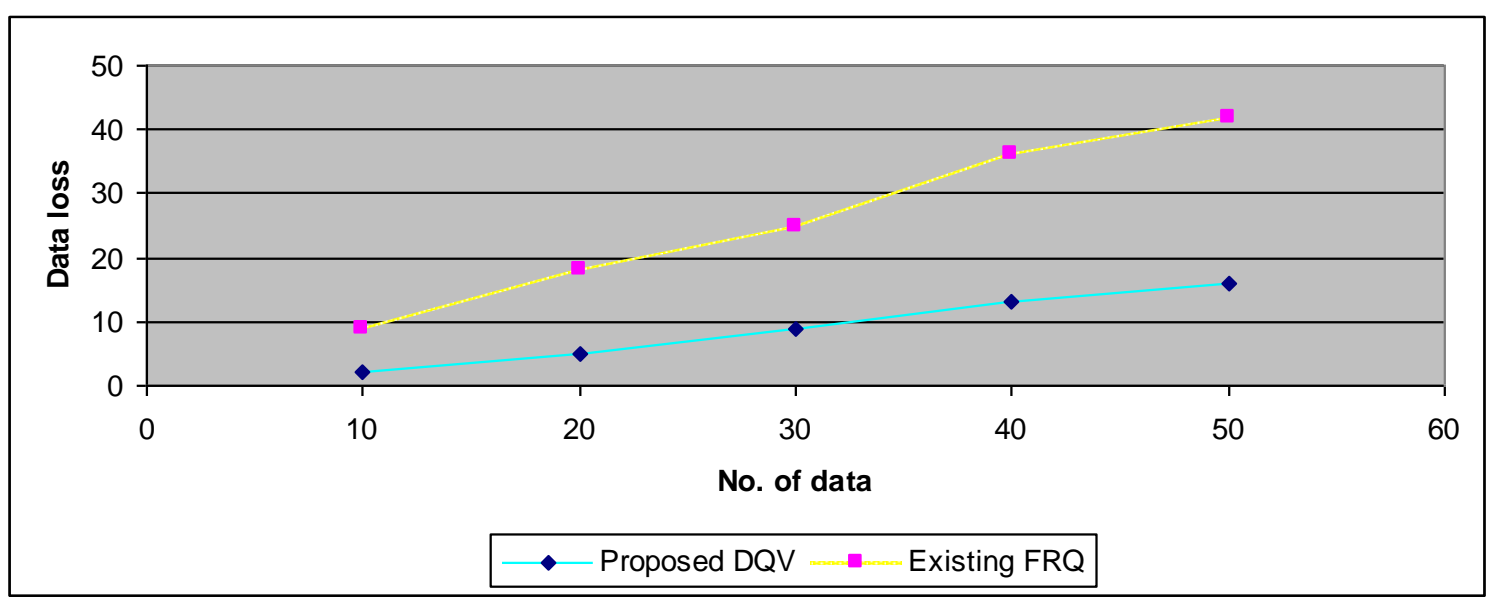

Fig 5. No. of data vs. Data loss 


\section{CONCLUSION}

The proposal work presented Deployment of Query Validation Scheme for Detecting and validating the Mobile Adversary Replica Nodes in Wireless Sensor Networks in which it expands replica detection method by validation with FRQ technique to detecting the mobile replica nodes and discards the varying query choices of mobile sensor nodes. The proposed Query Validation Scheme reduces the message query transmission by discarding the query based on its validity. We performed simulations of the proposed Query Validation Scheme under an arbitrary association attack strategy under a static assignment attack approach in which he maintains his replicas from moving to best detection. The results of these simulations given that the proposed Query Validation Scheme rapidly validates mobile replicas with a small amount of position claims beside each approach.

\section{REFERENCES}

[1] J. Ho, M. Wright, and S. K. Das, "Fast Detection of Mobile Replica Node Attacks in Wireless Sensor Networks Using Sequential Hypothesis Testing", IEEE TRANSACTIONS ON MOBILE COMPUTING, VOL. 10, NO. 6, JUNE 2011.

[2] Zhenzhen Ye and Alhussein A. Abouzeid, "Optimal Stochastic Location Updates in Mobile Ad Hoc Networks", IEEE TRANSACTIONS ON MOBILE COMPUTING, VOL. 10, NO. 5, MAY 2011

[3] H. Liu, L. Selavo, J. Stankovic , "SeeDTV: Deployment-Time Validation for Wireless Sensor Networks", EmNets'07, June 25-26, 2007, Cork, Ireland

[4] Byun, H. et. Al., "Adaptive Duty Cycle Control with Queue Management in Wireless Sensor Networks", IEEE Transactions on Mobile Computing, 2012
[5] M. Dyer, J. Beutel, 'Deployment Support Network -A Toolkit for the Development ofWSNs. In Fourth European Workshop on Sensor Networks, 2007.

[6] Durmaz Incel, O. et. Al., "Fast Data Collection in Tree-Based Wireless Sensor Networks", IEEE Transactions on Mobile Computing, Jan. 2012

[7] Chih-Kuang Lin et. Al., "A Distributed and Scalable Time Slot Allocation Protocol for Wireless Sensor Networks", IEEE Transactions on Mobile Computing, April 2011

[8] J. Li, Y. Bai, et. Al., . POWER: Planning and Deployment Platform for Wireless Sensot Networks. In Fifth International Conference on Grid and Cooperative Computing Workshops, 2006.

[9] Rui Tan et. Al., "Exploiting Reactive Mobility for Collaborative Target Detection in WirelessSensorNetworks", IEEE Transactions on Mobile Computing, march 2010

[10] Shigeng Zhang et. Al., "Accurate and Energy-Efficient Range-Free Localization for Mobile Sensor Networks", : IEEE Transactions on Mobile Computing, June 2010

[11] Xue Wang et. Al., "Distributed Energy Optimization for Target Tracking in Wireless Sensor Networks", IEEE Transactions on Mobile Computing, Jan 2010

[12] Jiang, B. et. Al., "Probability-based Prediction and Sleep Scheduling for Energy Efficient Target Tracking in Sensor Networks",

IEEE Transactions on Mobile Computing, 2012 